\title{
Equation of state for hard-square lattice gases
}

\author{
Heitor C. Marques Fernandes, ${ }^{*}$ Yan Levin, ${ }^{\dagger}$ and Jeferson J. Arenzon ${ }^{*}$ \\ Instituto de Física, Universidade Federal do Rio Grande do Sul, Caixa Postale 15051, 91501-970 Porto Alegre RS, Brazil
}

(Received 20 November 2006; revised manuscript received 23 February 2007; published 18 May 2007)

\begin{abstract}
A simple equation of state is derived for a hard-core lattice gas of side length $\lambda$, and compared to the results of Monte Carlo simulations. In the disordered fluid phase, the equation is found to work very well for a two-dimensional lattice gas of hard squares and reasonably well for the three-dimensional gas of hard cubes.
\end{abstract}

DOI: 10.1103/PhysRevE.75.052101

Almost forty years ago, Carnahan and Starling published their now famous equation of state for a hard-sphere fluid [1]. Their derivation was based on the simple observation that the leading order virial coefficients for a hard-sphere fluid in three dimensions closely followed a geometric sequence. The assumption that this behavior also extrapolated to higher-order virials allowed Carnahan and Starling to explicitly resum the virial expansion to find a simple, yet very accurate, equation of state.

Unfortunately, no such accurate equation of state is known for the case of lattice gases. This is particularly frustrating, since lattice models are widely used to study many complex fluids ranging from microemulsions to electrolytes [2-7]. In this Brief Report, we shall present a very simple equation of state, which works very well for a twodimensional lattice gas of hard squares and reasonably well for a three-dimensional lattice gas of small hard cubes at not too high density.

Our discussion is based on a lattice theory of polymer mixtures proposed a long time ago by Flory [8], who deduced the entropy of mixing to be

$$
S=-k_{B}\left(N_{1} \ln \phi_{1}+N_{2} \ln \phi_{2}\right),
$$

where $N_{1}$ and $N_{2}$ are the numbers of polymers of types 1 and 2 , while $\phi_{1}$ and $\phi_{2}$ are their respective volume fractions. The form of Eq. (1) is particularly appealing since it does not contain any reference to the lattice structure and depends only on thermodynamically well-defined variables. The mixture is assumed to fill all the available volume, so that there are no vacancies. If there is only one type of polymer occupying a volume fraction $\phi_{1}$, the rest of the space is taken to be filled by the solvent of $\phi_{2}=1-\phi_{1}$.

It is clear that the formalism developed by Flory for polymer mixtures should be readily applicable to "hard" nonattracting lattice gases. Consider, for example, a lattice gas of hard hypercubes of volume $\lambda^{d}$ (the lattice spacing is taken to be 1 ) on a simple hypercubic lattice in $d$ dimensions. ${ }^{1}$ The

\footnotetext{
*Electronic address: heitor@if.ufrgs.br

${ }^{\dagger}$ Electronic address: levin@if.ufrgs.br

"Electronic address: arenzon@if.ufrgs.br

${ }^{1}$ Notice that the formalism developed below works well only when $\lambda$ is commensurate with the lattice spacing. It does not apply for systems of tilted squares, whose length can be noninteger [10].
}

Helmholtz free energy of this lattice gas is $F_{m}=-T S$, since the system is athermal. The free energy density $f_{m}=F_{m} / V$ is

$$
\beta f_{m}=\rho \ln \phi+(1-\phi) \ln (1-\phi),
$$

where $\beta=1 / k_{B} T, \rho$ is the particle density, and $\phi=\lambda^{d} \rho$ is the volume fraction.

We note, however, that in the low-density limit, Eq. (2) does not reduce to the free energy of the ideal gas

$$
\beta f=\rho \ln \rho-\rho .
$$

Therefore, $f_{m}$ cannot be the total free energy of the system, except for the case of $\lambda=1$ when Eq. (2) becomes exact. For polymer mixtures, to obtain the total free energy, Flory added an extra contribution to Eq. (2) which accounted for the conformational degrees of freedom of polymer chains, the so-called entropy of disorientation [8]. This restored the correct low-density behavior to the theory. For rigid particles, however, the entropy of disorientation is identically zero and cannot be the reason for the failure of Eq. (2).

To recover the correct low-density behavior, while preserving the simple and thermodynamically appealing form of Eq. (2), we modify $f_{m}$ by introducing a multiplicative factor $g(\lambda)$ into Eq. (2),

$$
\beta f=\rho \ln \phi+g(\lambda)(1-\phi) \ln (1-\phi) .
$$

This equation can be interpreted as an interpolation between the low-density limit governed by the particles, and the high-

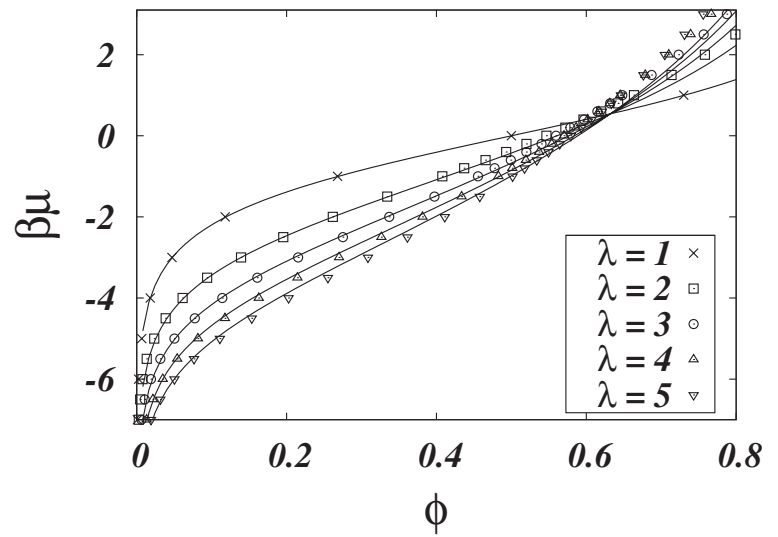

FIG. 1. Chemical potential versus volume fraction for various two-dimensional lattice gases. The symbols are the Monte Carlo (MC) results while the lines are the predictions of the MFA, Eq. (6), with $d=2$. 


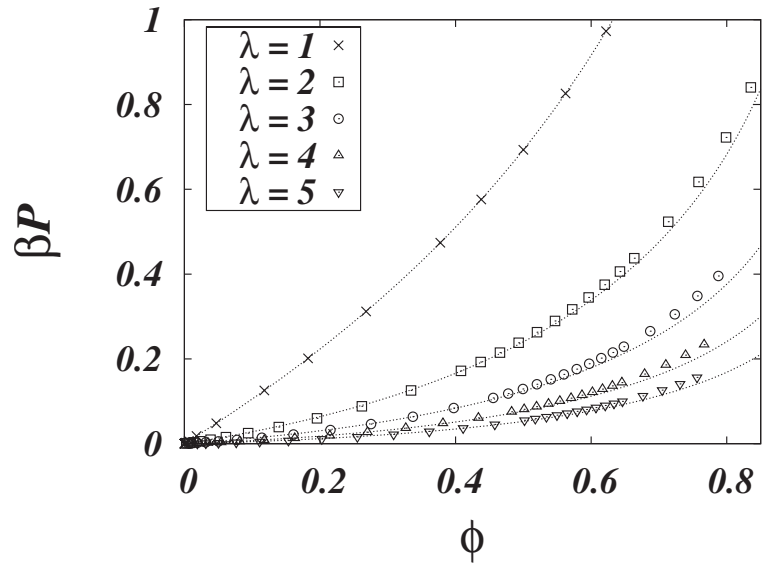

FIG. 2. Pressure versus volume fraction for various twodimensional lattice gases. The symbols are obtained from the MC results by integrating the Gibbs-Duhem equation, while the lines are the predictions of the MFA, Eq. (7), with $d=2$.

density limit in which defects, the "holes," become relevant. The total number of holes, however, is not fixed, since the vacancies can change their size and shape, so that the prefactor appearing in front of the second term of Eq. (4) is the effective number density.

The requirement that in the low-density limit Eq. (4) must reduce to Eq. (3) uniquely determines the functional form of $g(\lambda)$, yielding

$$
g(\lambda)=\frac{1+d \ln \lambda}{\lambda^{d}}
$$

Note that $g(1)=1$, so that Eq. (4) reduces to the exact free energy for the lattice gas of $\lambda=1$. The chemical potential $\mu$ $=\partial F / \partial N$ within the modified Flory approximation (MFA) is

$$
\beta \mu=-(1+d \ln \lambda)[\ln (1-\phi)+1]+\ln \phi+1,
$$

while the pressure $P=-\partial F / \partial V$ is

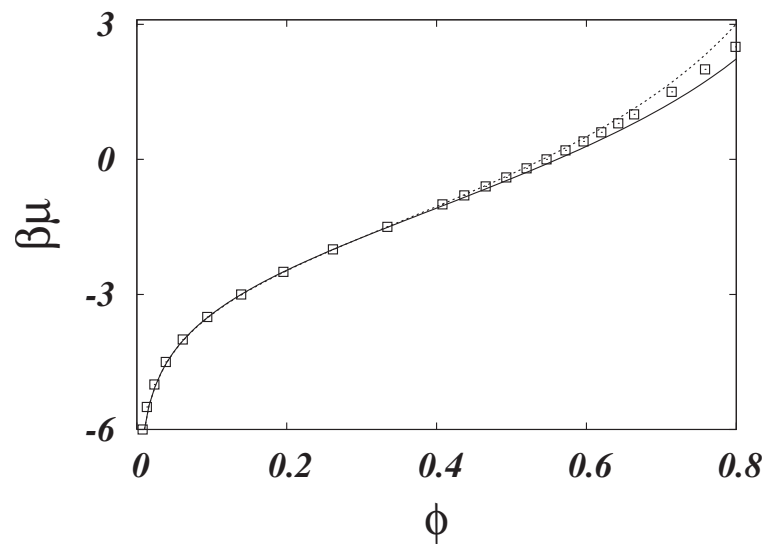

FIG. 3. Chemical potential versus volume fraction for $\lambda=2$ lattice gas. The symbols are the MC results; the solid curve is the equation of state Eq. (6); the dashed line is the equation of state obtained using the fundamental measure density functional theory, Ref. [13], which is also the same as the one found earlier by Temperley [12]. The order-disorder transition occurs at $\phi \approx 0.93$ [10].

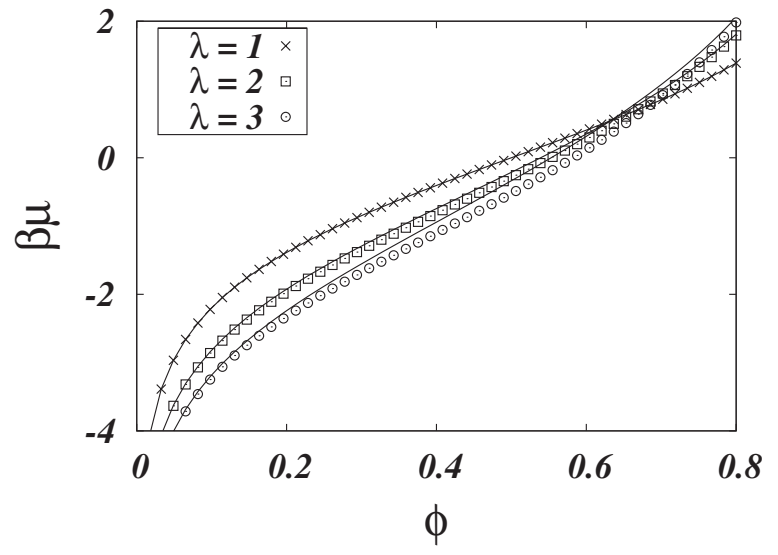

FIG. 4. Chemical potential versus volume fraction for a gas of hard rods with $\lambda=1,2$, and 3 in $d=1$. The symbols are the exact value of the chemical potential [14] while the lines are the predictions of the MFA, Eq. (6), with $d=1$.

$$
\beta P=\rho-g(\lambda)[\ln (1-\phi)+\phi] .
$$

In Fig. 1 we compare the value of the chemical potential obtained within the MFA with the results of the Monte Carlo simulations for a gas of hard squares of different sizes $\lambda$. The simulations were performed using the grand-canonical ensemble at fixed volume $V$, temperature $T$, and chemical potential $\mu$, with trial moves the insertion and removal of particles as well as attempts to diffuse [9]. The agreement is excellent for all $\lambda$ 's tested, up to quite high volume fractions. The Monte Carlo pressure can be obtained by integrating the Gibbs-Duhem equation, which in the athermal case reduces to $d \mu=\rho^{-1} d P$, with the low-density reference state given by the ideal gas. The agreement is again very good, as can be seen from Fig. 2. However, similarly to the CarnahanStarling equation of state, the MFA also fails to notice the phase transition between the disordered and ordered (columnar) phases present at high volume fractions $[10,11]$. In Fig. 3 we compare the accuracy of the MFA with the earlier equation of state derived by Temperley [12], which is also identical to the one recently found using the fundamental mea-

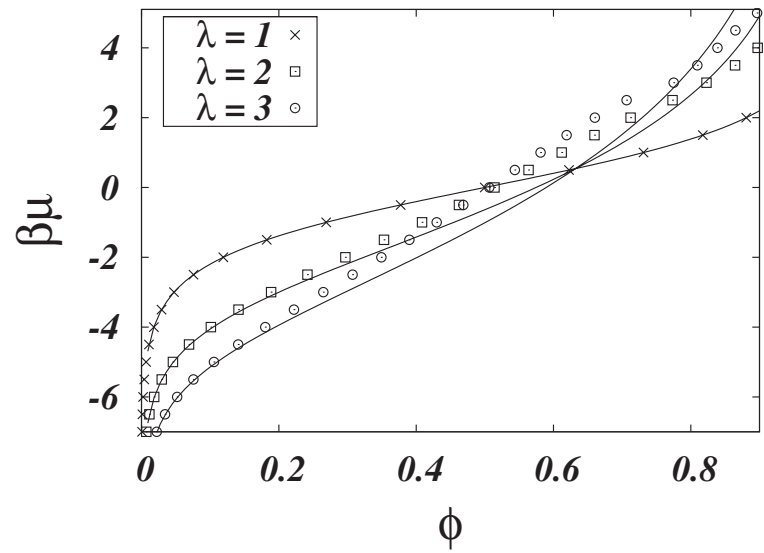

FIG. 5. Chemical potential versus volume fraction for various three-dimensional hard-cube lattice gases. The points are the MC results while the lines are the predictions of the MFA, Eq. (6), with $d=3$. 
sure theory [13]. We see that the simple equation of state derived in the present paper is of the same degree of accuracy as the ones obtained using more sophisticated methods. One advantage of our equation of state, however, is that it explicitly gives the dependence on the size of the particles. This is not the case for more sophisticated theories which yield equations of state that become progressively more complex for larger values of $\lambda$ without an apparent gain in accuracy.

It is curious that all the $\mathrm{MC}$ curves for different values of $\lambda$ intersect at approximately the same point. This property is also captured by the MFA, which predicts that all the chemical potentials for different $\lambda$ 's are equal when the volume fraction satisfies $\ln \left(1-\phi_{\times}\right)=-1$, independent of $d$. The value $\phi_{\times}=0.632121$ is in excellent agreement with the intersection point observed in the Monte Carlo simulations.
In Figs. 4 and 5 we also show the equations of state for $d=1$ hard rods, and $d=3$ hard cubes. In the case of $d=1$ the exact free energy is known [14]. Although still quite good, the agreement between the simulations and the MFA deteriorates more rapidly with increasing $\lambda$ for $d=1$ and $d=3$ than for $d=2$.

The high degree of accuracy of the MFA in $d=2$ is quite surprising in view of the crudeness of the approximation. It also suggests that there should be a more direct way to arrive at Eq. (4), or some other such equation of state $[13,15]$, generally valid for nonattracting lattice gases of arbitrary $\lambda$. In the absence of such a general theory, the very simple Eq. (4) should be useful for constructing lattice mean-field theories for various complex systems.

This work was supported in part by the Brazilian science agencies CNPq, CAPES, and FAPERGS.
[1] N. F. Carnahan and K. E. Starling, J. Chem. Phys. 51, 635 (1969).

[2] J. Marro and R. Dickman, Nonequilibrium Phase Transitions in Lattice Models (Cambridge University Press, Cambridge, U.K., 1999).

[3] B. Widom, J. Chem. Phys. 81, 1030 (1984).

[4] E. Caglioti and V. Loreto, Phys. Rev. Lett. 83, 4333 (1999).

[5] M. N. Artyomov, V. Kobelev, and A. B. Kolomeisky, J. Chem. Phys. 118, 6394 (2003).

[6] A. Panagiotopoulos, J. Chem. Phys. 123, 104504 (2005).

[7] M. Tarzia et al., Europhys. Lett. 66, 531 (2004).

[8] P. J. Flory, Principles of Polymer Chemistry (Cornell University Press, Ithaca, NY, 1953).
[9] D. Frenkel and B. Smit, Understanding Molecular Simulation (Academic Press, New York, 2002).

[10] H. C. M. Fernandes, J. J. Arenzon, and Y. Levin, J. Chem. Phys. 126, 114508 (2007).

[11] L. K. Runnels, in Phase Transitions and Critical Phenomena, edited by C. Domb and M. S. Green (Academic Press, London, 1972), Vol. 2, Chap. 8, pp. 305-328.

[12] H. Temperley, Proc. Phys. Soc. London 77, 630 (1961).

[13] L. Lafuente and J. A. Cuesta, J. Chem. Phys. 119, 10832 (2003).

[14] A. Robledo, J. Chem. Phys. 72, 1701 (1980).

[15] L. Lafuente and J. A. Cuesta, Phys. Rev. Lett. 93, 130603 (2004). 